Recherches en didactique des langues et des cultures

Les cahiers de l'Acedle

7-2| 2010

Les langues tout au long de la vie: Permanences et évolutions en didactique des langues

\title{
Reconnaissance des parcours individuels dans une méthodologie de groupe
}

Sophie Dufour et Chantal Parpette

\section{(2) OpenEdition}

Journals

Édition électronique

URL : https://journals.openedition.org/rdlc/2119

DOI : $10.4000 /$ rdlc. 2119

ISSN : 1958-5772

Éditeur

ACEDLE

Référence électronique

Sophie Dufour et Chantal Parpette, «Reconnaissance des parcours individuels dans une

méthodologie de groupe », Recherches en didactique des langues et des cultures [En ligne], 7-2 | 2010,

mis en ligne le 01 octobre 2010, consulté le 15 février 2023. URL : http://journals.openedition.org/rdlc/ 2119 ; DOl : https://doi.org/10.4000/rdlc.2119

Ce document a été généré automatiquement le 29 septembre 2020.

\section{(i) 8}

Creative Commons - Attribution - Pas d'Utilisation Commerciale - Pas de Modification 4.0 International - CC BY-NC-ND 4.0

https://creativecommons.org/licenses/by-nc-nd/4.0/ 


\title{
Reconnaissance des parcours individuels dans une méthodologie de groupe
}

\author{
Sophie Dufour et Chantal Parpette
}

\section{Introduction}

1 Tout apprenant de langue étrangère est porteur d'habitudes d'apprentissage, liées à des apprentissages antérieurs, qui influencent son approche de la formation linguistique. À cela s'ajoutent les raisons qui l'amènent à entreprendre cet apprentissage, ses habitudes culturelles et ses goûts personnels. Les diversités que cela introduit dans un groupe d'apprenants sont particulièrement nettes dans le contexte des centres de langues en France, dont la spécificité est de réunir des publics à la fois adultes et de différentes origines géographiques. Considérant que ce contexte d'enseignement, qui est le nôtre, constitue un espace privilégié d'interrogation et d'expérimentation, nous avons entrepris en octobre 2009, auprès de groupes d'apprenants de niveau B1, une recherche-action intitulée "Parcours individuels dans une méthodologie de groupe".

2 Nous avons eu l'occasion de montrer antérieurement comment l'enseignement du FLE en France utilise relativement peu le potentiel qu'offre le contexte linguistique et institutionnel dans lequel il opère (Parpette, 2006), et comment il serait possible de faire évoluer la méthodologie d'enseignement vers une intégration du milieu environnant. Cette réflexion a été mise en œuvre dans la réalisation de la méthode Ici qui combine l'apprentissage en classe et l'apprentissage dans le milieu extérieur à travers observations, enquêtes et rencontres (Abry et al., 2006). La recherche-action exposée ici poursuit cette réflexion d'élargissement de la classe et de son enseignement collectif, en s'intéressant plus spécifiquement aux profils individuels des étudiants dans l'élaboration du programme d'enseignement. 


\section{Notions didactiques en jeu}

3 La méthodologie communicative, avec la notion d'analyse des besoins et plus encore celle de centration sur l'apprenant, offre dans son principe un cadre théorique permettant de penser la mise en œuvre d'un enseignement-apprentissage adapté à cette réalité des centres de FLE en France.

Les orientations méthodologiques et les matériels proposés doivent être de mieux en mieux ciblés, c'est-à-dire de mieux en mieux adaptés à la spécificité et aux besoins (en matière de pratique de la communication) de groupes d'apprenants précis. (Boyer, Butzbach \& Pendanx, $1990: 12$ )

C'est ainsi qu'était définie l'approche communicative, qui, au début des années 70, tendait à s'imposer. Cette prise en compte des besoins du public "en matière de pratique de la communication" a d'abord signifié une prise en charge dans les programmes et le matériel d'enseignement de situations de communication et de types de discours alignés sur les objectifs d'apprentissage des apprenants : insertion dans la vie quotidienne française, vie professionnelle au contact de francophones, etc., avec une détermination plus ou moins précise des situations langagières en cause. Mais au fil du temps, le terme de besoins a recouvert des paramètres de plus en plus variés. Parti d'un recensement des situations de communication, destiné à opérer une sélection d'actes de parole et de structures linguistiques à enseigner, il s'est progressivement étendu aux cultures scolaires et aux habitudes d'apprentissage (Pochard, 1994 ; Beacco et al., 2005). Richterich (1986) en avait très tôt montré le foisonnement superposant les besoins objectifs aux désirs personnels, et par là une certaine difficulté à intégrer les "besoins" de manière opérationnelle dans le quotidien de la classe.

La centration sur l'apprenant vient compléter cette perspective, en l'orientant davantage sur la prise en charge du profil personnel des apprenants, goûts, modes d'apprentissage, etc. Mais cette centration sur l'apprenant, intellectuellement et pédagogiquement séduisante a priori, pose d'épineuses questions lorsque l'on envisage concrètement sa mise en œuvre. De quel apprenant parle-t-on à travers ce singulier qui, en français, désigne autant un collectif qu'un individu? S'il s'agit de l'apprenant-groupe, cette centration ne présente pas de difficulté majeure. Elle consiste à prendre en compte les paramètres les plus explicitement communs à tous les membres du groupe : le niveau de connaissance linguistique, la langue et la culture d'origine, quand une nationalité est fortement représentée dans le groupe, les objectifs d'apprentissage quand ils sont partagés (ce qu'on appelle souvent les "programmes spécifiques"). Il existe tout d'abord le versant institutionnel du principe de centration, par le traitement spécifique de publics faisant l'objet d'accords bilatéraux entre universités étrangères et françaises, et pour les étudiants "individuels" par le biais des groupes de niveaux, qui ont toujours existé, qu'on les nomme "débutant", "intermédiaire" ou A1, B1, etc. On peut y ajouter l'organisation des cours, qui permet aux apprenants de choisir des options ou des modules en fonction de ce qui les intéresse. Cette centration se décline également sur le mode didactique dans les démarches d'enseignement choisissant les thèmes de travail liés aux centres d'intérêts manifestés par les apprenants, leur faisant mener des enquêtes sur des sujets de leur choix, etc. Les exemples pourraient être multipliés.

Mais la question se pose d'une tout autre manière dès lors que sous le terme apprenant, il est question de chaque individu membre du groupe. Comment prendre en compte des 
désirs, des goûts particuliers en matière de contenus traités, des habitudes de travail différentes, des objectifs qui ne se croisent pas entre les apprenants "de passage" pour un semestre de langue et ceux qui se destinent à un cursus universitaire en France, par exemple? Comment concilier le goût pour les apprentissages plus informels de certains et le besoin d'encadrement plus porté sur l'activité scolaire et écrite des autres? Cette vision individualisée de la centration sur l'apprenant apparaît difficilement compatible avec les conditions d'un enseignement collectif en milieu institutionnel, centré autour du groupe et des paramètres communs à tous les individus qui le composent. Ces observations ne constituent pas un aveu d'impuissance, mais simplement le constat d'une tension entre organisation collective et attention aux parcours individuels. Si nous partageons l'avis que "la centration sur l'apprenant reste largement à construire conceptuellement" (Cuq, 2003: 40), la notion nous parait productive, surtout dans le contexte des centres de FLE en France, et c'est à cette construction que nous essayons de contribuer dans cette recherche-action.

7 Une autre notion est venue plus récemment s'ajouter à ces principes: celle d'enseignement-apprentissage en contexte homoglotte, proposée à la réflexion des didacticiens au moment, entre autres, du colloque de l'Adcuefe de 2005 (Fievet \& Abry, 2006). Nous avions à cette occasion évoqué la relative indifférenciation qui prévaut la plupart du temps entre l'enseignement du français en pays d'origine d'une part, et en contexte francophone, d'autre part. Le milieu environnant en contexte homoglotte offre pourtant des ressources immenses sur différents plans, qui pourraient constituer le support d'une véritable méthodologie d'enseignement jouant sur l'aller-retour entre la classe et l'extérieur, dont nous avons décrit l'une des déclinaisons possibles (Parpette, 2006). Au-delà de son intérêt pour tout enseignement de FLE, l'ouverture de la classe sur le milieu extérieur représente un des moyens de particulariser l'enseignement collectif en permettant à chaque apprenant de choisir d'y travailler sur l'aspect de son choix, comme on le verra plus loin.

\section{Démarches et activités}

8 La recherche-action présentée ici intègre donc le principe de centration sur l'apprenant et la réflexion sur l'apprentissage en milieu homoglotte. Elle concerne un groupe de 14 étudiants (10 filles, 4 garçons ; 2 Japonaises, 1 Suédoise, 11 Chinois) de cours intensifs (280h réparties sur 12 semaines) de niveau B1. Les activités ont été mises en œuvre par deux enseignantes à raison de $10 \mathrm{~h} 30$ hebdomadaires.

9 La prise en compte des parcours individuels repose sur les 5 principes suivants mis en œuvre à travers un ensemble de procédures et d'activités :

\subsection{Connaissance des profils individuels : les entretiens}

10 Un enseignant ne dispose généralement que d'informations limitées concernant les apprenants de sa classe : leurs noms et leurs nationalités. La première étape consiste à en savoir un peu plus sur le profil individuel de chacun dans le groupe, condition pour pouvoir introduire dans le programme des propositions d'activités d'apprentissage plus individualisées. Des entretiens sont ainsi effectués au tout premier cours, les enseignantes interrogeant des binômes d'apprenants durant une vingtaine de minutes. Bien qu'assez souple, la trame de ces échanges vise à obtenir des informations sur 
l'identité, le cursus scolaire et éventuellement professionnel de chaque étudiant, mais aussi sur ses goûts personnels, les objectifs de son apprentissage du français, ses formes de travail habituelles, les compétences qu'il souhaite développer, les thèmes qu'il aimerait aborder dans le programme. Certains sont là pour poursuivre des études en France (dans la mode, le commerce, le droit), d'autres pour parfaire leur connaissance de la langue. Pour les premiers, les souhaits s'orientent naturellement vers leurs spécialités, les autres se révélant plus curieux d'aborder des sujets culturels, d'aller à la rencontre des Français. Par ailleurs, et de manière assez cohérente avec le contexte homoglotte, les demandes sur le plan langagier sont très nettement orientées vers les interactions orales.

\subsection{Individualisation des rythmes de travail : activités en sous- groupes}

11 Le travail en grand groupe, surtout à l'oral, n'est pas particulièrement favorable au respect du rythme d'apprentissage de chacun : il met en effet plutôt en avant les échanges enseignant-étudiants, et n'a pas les moyens d'assurer la participation effective de chacun au sein d'une activité. Nous avons donc opté pour accentuer le travail en sous-groupes. $\mathrm{Si}$ cette pratique est relativement répandue en FLE, nous en avons renforcé les effets par la présence de stagiaires venant des filières FLE. Six d'entre eux ont participé régulièrement aux cours et autres activités, ce qui a permis de constituer des sous-groupes réunissant 2 à 3 étudiants et 1 stagiaire. Outre le fait qu'elle tend à favoriser naturellement la multiplication du volume de parole de chacun et aide au développement de la fluidité, la communication en sous-groupe permet à chaque individu de s'exprimer, non seulement sur le plan linguistique mais aussi personnel, chacun selon ses modalités propres. La présence d'un stagiaire dans le groupe dynamise la communication et démultiplie la fonction enseignante. Les activités guidées (concertation-correction d'une compréhension orale par exemple) prennent aussi souvent un caractère largement conversationnel, du fait du cadre participatif restreint et de l'âge des stagiaires assez proche de celui des apprenants, ce qui favorise une dynamique dialogale dans laquelle les statuts, rôles et places interactionnelles se superposent et s'imbriquent volontiers (Vasseur, 2005). On remarquera au passage que l'on se retrouve dans une situation de communication proche de celle observée en cours particuliers de langue, où conversation exolingue et interaction didactique s'harmonisent dans l'interaction (Dufour, 2007).

\subsection{Ouverture de la classe sur l'extérieur : les projets culturels et langagiers}

12 La prise en compte des goûts et des désirs de contacts avec l'environnement humain et culturel confirmés durant les entretiens se traduit par la mise en œuvre de projets sur la ville. Le contexte homoglotte ouvre des possibilités très riches de combinaison d'apprentissage entre la classe et le milieu extérieur. La mise en place d'activités 
extérieures sur des thématiques choisies lors des entretiens (sport, cuisine, mode, etc.) s'est alignée sur les demandes des étudiants.

Il s'agissait donc de construire des projets par petits groupes de deux ou trois apprenants axés sur la vie des Français, et plus particulièrement des Lyonnais. Parmi les sujets choisis on retiendra :

- Les Berges du Rhône (transformation des parkings le long du Rhône en une zone de promenade et de loisirs de $6 \mathrm{~km}$ ) ;

- Les Halles Paul Bocuse de Lyon (marché abrité regroupant des stands de produits alimentaires de qualité);

- Le Village des Créateurs ${ }^{1}$ (quartier qui concentre une pépinière de jeunes entreprises de mode à Lyon).

Les projets consistent à rechercher des informations sur internet, à prendre des contacts avec les acteurs concernés (commerçants, usagers, etc.), à les interviewer, à prendre des photos. Ils aboutissent à différentes formes de réalisation finale: compte-rendu oral accompagné d'un diaporama en classe, ou organisation d'une visite guidée pour le reste du groupe.

15 Ainsi, pour la visite des Halles Paul Bocuse, le groupe responsable du projet a organisé des activités sur place avec le reste de la classe. Il a tout d'abord présenté son travail dans un café, expliquant les spécialités gastronomiques locales que les autres étudiants devaient ensuite retrouver afin de remplir un questionnaire au cours de la visite organisée. Ces derniers avaient aussi reçu pour consigne de demander des explications aux commerçants sur les modes de préparation de certains plats typiques. L'activité s'est ensuite déroulée dans les Halles, encadrée et guidée par les responsables du projet, une enseignante et une stagiaire.

16 Ces activités ne se situent pas en marge de l'enseignement en classe. Elles développent les compétences prévues par le programme: à l'oral, interview et exposé, à l'écrit, questionnaire et synthèse, tout en favorisant l'épanouissement des individualités au sein du groupe.

\subsection{Valorisation des ressources individuelles : le SEL}

Les principes qui précèdent sont mis en œuvre dans des procédures ou activités relevant du format habituel de l'enseignement collectif, qu'il s'agisse de l'organisation du travail en petits groupes ou des recherches à l'extérieur de la classe. Le point évoqué ici concerne les savoir-faire des étudiants indépendamment de tout lien avec l'apprentissage des langues. Il s'agit de la mise en place d'un $\mathrm{SEL}^{2}$, Système d'Échange Local ou Service d'échange Local. C'est un regroupement de personnes qui pratiquent l'échange de biens, de services et de savoirs. On peut échanger par exemple une heure d'utilisation d'un piano contre une heure d'initiation à l'informatique, ou encore une invitation à déjeuner contre du jardinage.

18 Nous avons tenté l'expérience avec 21 personnes (13 étudiants, 2 enseignants et 6 stagiaires de Master FLE). Après avoir découvert les SEL à travers une activité de compréhension orale de documents vidéo sur internet ${ }^{3}$, les apprenants, avec l'aide des stagiaires, ont rempli des fiches d'offres et demandes sur des formulaires papier dont voici un exemple. 


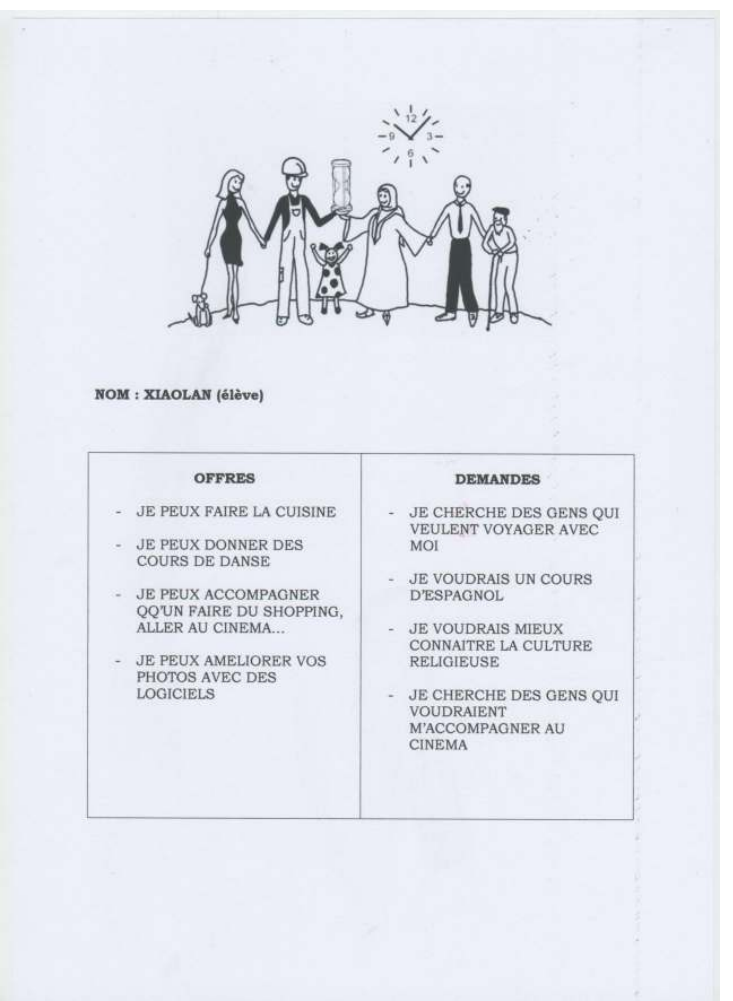

19 Une matinée SEL a ensuite été organisée durant laquelle les propositions de chaque participant ont été affichées au mur. Pour chaque échange, un contrat stipulant l'identité de chacun des deux participants, les objets de la transaction, les dates et lieux prévus pour les échanges a été signé par les deux parties.

Voici quelques exemples de contrats passés :

- un cours de chinois contre une séance de maquillage ;

- prêt de DVD contre un repas ;

- un cours de tricot contre un cours de suédois ;

- une visite au marché aux puces en voiture contre un repas.

Les retours sur cette activité sont très positifs et montrent un réel investissement de la part des étudiants, qui sont valorisés dans leur rôle de locuteur allophone, et se voient ainsi capables d'agir et d'échanger "naturellement" en langue cible. Certains confient qu'ils ont appris à se connaître différemment dans la classe en passant du temps ensemble lors de ces échanges, ce qui a selon nous fortement contribué à souder le groupe-classe. Ces activités se situent en-dehors du cadre institutionnel d'apprentissage de la langue. Elles ne donnent pas lieu à un compte-rendu et n'entrent dans aucun système d'évaluation, ce qui n'empêche pas une pratique intensive et spontanée du français, lorsque celui-ci est la seule langue commune du binôme. Elles répondent à des besoins ou à des désirs, élargissent le champ des relations entre les participants, permettent aux étudiants d'exprimer leurs talents, et de se définir au-delà de leur seul statut d'apprenant. 


\subsection{Réflexion sur l'apprentissage : bilan et engagement hebdomadaires}

Une prise en compte des profils variés présents dans la classe gagne à s'accompagner chez les étudiants d'une réflexion sur leurs stratégies d'apprentissage. Chaque semaine, les étudiants ont été invités à remplir des fiches de bilan-engagement. Constitués de 2 parties, celles-ci sont destinées dans un premier temps - partie bilan - à recenser les actions langagières ou culturelles faites pour apprendre la langue-cible, aussi bien en classe qu'à l'extérieur. Dans une seconde partie - fiche-engagement - les étudiants listent les actions qu'ils entendent programmer par eux-mêmes en dehors des cours.

Figure 2 - Fiche bilan.

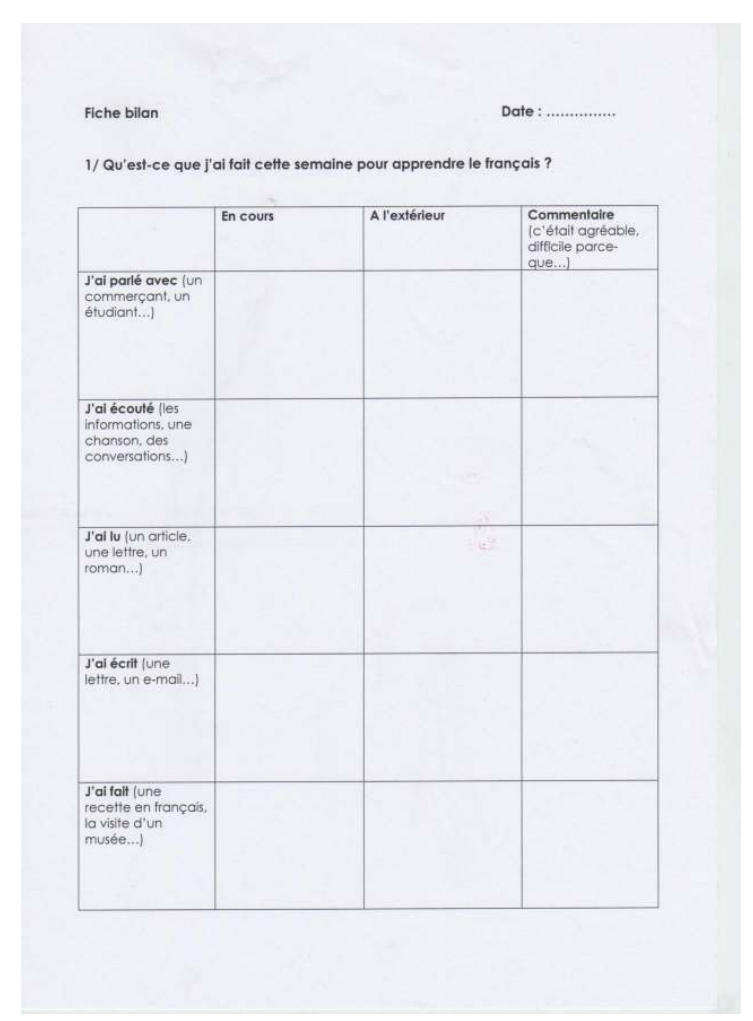


Figure 2 suite - Fiche engagement.

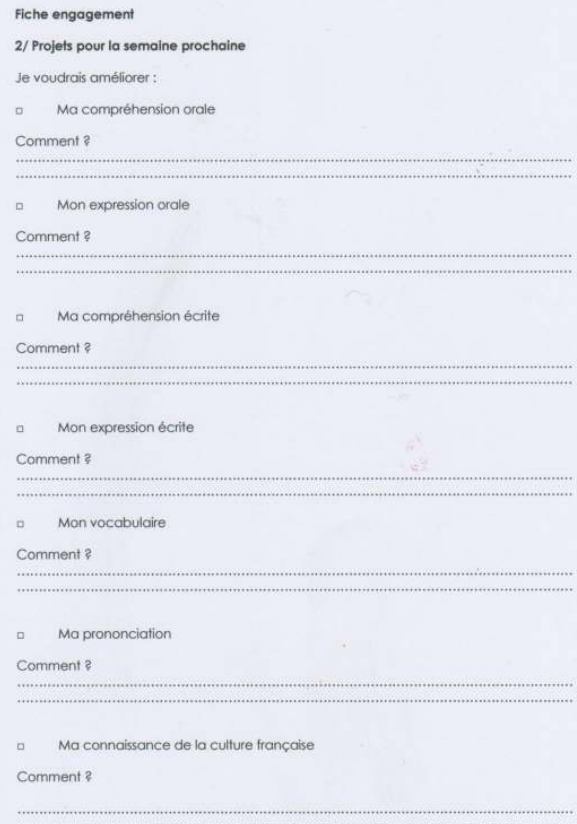

Les données apparaissant dans ces fiches apportent des informations intéressantes :

\section{Fiche bilan}

1/ Qu'est-ce que j'ai fait cette semaine pour apprendre le français? J'ai parlé avec :

1 :...deux coréennes. Je pense que mon françaises est meilleur qu'elles.

2 : ...des employés de SFR. Ils avaient un peu de mal, parce qu'ils ont tutoyer pour nous, les japonais. C'était dommage!

$3:$...un homme qui m'aidé avec le déménagement quelques fois pendant la semaine. C'était agréable parce qu'il sait que je ne parle pas français bien alors il parle lentement

Lorsqu'on invite les apprenants à s'exprimer sur l'activité interactionnelle qu'ils entretiennent dans leur milieu environnant quotidien, on constate l'existence d'une conscience réflexive. Ceux-ci s'expriment en effet facilement soit sur leur niveau en langue (1 et 3), soit sur les représentations que les natifs peuvent avoir d'eux en tant que locuteurs allophones (2).

J'ai écouté :

4 :...dans le tram, le métro, le bus. C'était bien, j'ai pu comprendre de quoi on a parlé, petit à petit

5:...une chanson qui s'appelle "comme toi". C'était agréable parce que elle avait beaucoup démotion

Ces deux témoignages montrent les initiatives prises par certains pour améliorer leur compétence en français, prenant appui sur la réalité de leur quotidien (4 et 5).

J'ai lu :

6 : ... un peu le "Petit Nicolas". Je peux comprendre sans le dictionnaire. 
$7:$ :... quelque lettre du France télécom et du EDF. C'est mauvais parce que je dois payer beaucoup et je n'ai pas compris le raison dit dans ces lettres

8 : ...une lettre à mon ancien propriétaire pour lui demander de me faire passer le courrier. C'était difficile

9 :...un mail à un ami français. C'était assez facile

Enfin, il nous semblait important de valoriser les actions qui mettent les apprenants en contact avec la langue, quelles qu'elles soient (10 et 11) :

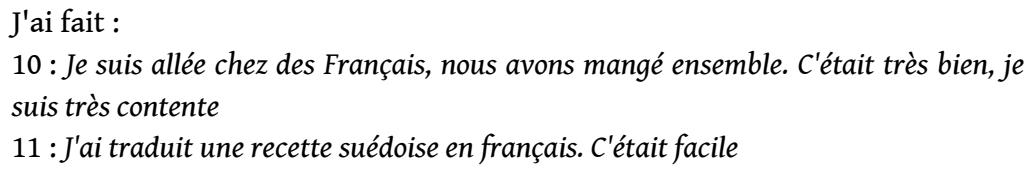

On peut faire l'hypothèse, à partir de ces témoignages, d'une prise de conscience de ce qui dans la vie quotidienne alimente naturellement l'apprentissage de la langue, prise de conscience qui peut ensuite donner lieu à des initiatives d'"engagements" telles que celles qui apparaissent dans ce qui suit.

\section{Fiche engagement}

2/ Projets pour la semaine prochaine :

1 : je regarderai les débats sur le site " $\mathrm{C}$ dans l'air"

2 : écouter mes colocs quand ils parlent

$3:$ je praticterai le future quan je parlerai

4 : je lirai un journal plus "qualitatif" que Lyon Plus

$5: j$ 'enverrai des e-mails pour mes amies françaises

$6: j$ 'écrirai mon journal intime

Et, concernant la connaissance de la culture française ...

$7:$ je vais un restaurant "Le Nord"

8 : j'irai de la musée de beaux-arts pour regarderai l'exposition de Picasso,

Monet, etc.

9 : aller aux puces

31 Au-delà des initiatives prises par les étudiants, ce dispositif de réflexivité fournit aux enseignants des données sur les manières de travailler des étudiants, et sur leurs dispositions à organiser leur apprentissage.

\section{Premier bilan et réflexions}

Cette première expérience fait naturellement émerger un certain nombre de réflexions et d'interrogations. 


\subsection{Entre le dedans et le dehors, les stagiaires} les filières de didactique du FLE. Ils peuvent ainsi offrir un premier terrain de stage aux étudiants de FLE. Lors de notre recherche, ceux-ci ont été largement intégrés au projet, comme on l'a vu plus haut, et y ont joué un rôle majeur, en particulier dans la constitution des petits groupes de travail dans lesquels ils remplissent une double fonction, celle d'enseignants accompagnant l'apprentissage en animant les moments d'échanges oraux, en expliquant, en corrigeant, et celle d'étudiants français discutant avec des étudiants étrangers. Ce double rôle donne un caractère particulier aux interactions, qui alternent entre activité de langue et discussion ordinaire avec de jeunes Français, intéressante combinaison entre le contenu et la forme, entre le rôle d'apprenant de langue et celui d'étranger résidant en France.

\subsection{Apprentissage programmé, apprentissage informel}

Une première interrogation concerne la compatibilité entre l'apprentissage lors des communications extérieures à la classe et le programme d'enseignement fixé par l'équipe pédagogique pour chaque niveau. Certains aspects de ce programme s'intègrent sans difficulté aux activités extérieures. Dès lors en effet que les objectifs sont exprimés en termes larges d'aptitudes (compréhension orale, lecture, interaction orale), on imagine aisément comment l'expression orale peut trouver sa place hors de la classe, au moment des enquêtes, des rencontres diverses. Cela devient plus délicat en revanche lorsqu'il s'agit de répondre à des objectifs exprimés en termes grammaticaux par exemple. Les interactions naturelles hors de la classe ne connaissent ni catégorisation, ni niveau, ni progression, et n'offrent pas la prévisibilité des documents de travail construits pour la classe. Cela ne signifie pas qu'il n'y a pas d'apprentissage, bien au contraire, mais ces apprentissages ne sont pas vraiment prévisibles. Il est donc important d'envisager une manière de prendre en compte dans l'évaluation ce que les étudiants apprennent à l'extérieur. Comme le montrent les travaux de Stauber (2009), cela peut se faire à travers des procédures assez classiques de comptes-rendus, d'exposés sur les expériences extérieures, ou, de manière plus intéressante souvent, sous forme d'échanges entre les groupes comparant leurs expériences. Elle évoque également des moments d'autoréflexivité, au cours desquels les étudiants explicitent ce qu'ils ont appris sur le plan linguistique, des expressions nouvelles, des manières de dire les choses, etc.

\section{3. Évolution des habitudes de travail}

Cette individualisation de l'enseignement, si elle vise un objectif positif pour les apprenants, peut également les confronter à des ruptures par rapport à leurs habitudes et leurs représentations de l'apprentissage. Certains changements, tels que le travail en groupe avec des stagiaires français, rencontrent une adhésion quasiment sans réticence, d'autres en revanche peuvent demander un certain temps d'adaptation, et exigent d'être solidement encadrés. C'est le cas des projets sur la ville, qui doivent d'une part être accompagnés en classe, étape par étape (par exemple phase de recherche des premières informations sur internet, phase de rencontre avec les interlocuteurs ciblés, phase de 
rédaction ou de préparation du Powerpoint) et, d'autre part, être intégrés à l'évaluation pour être légitimés.

\subsection{Reconnaissance institutionnelle des activités hors classe}

Nous signalions dans la première partie de l'article la prégnance du format "classe" dans la conception de l'enseignement, y compris dans ces lieux de plus grande liberté et d'innovation que sont les centres de langues détachés du système éducatif. Les activités extérieures posent la question de leur reconnaissance par l'institution et les étudiants eux-mêmes, et du moyen de les intégrer techniquement dans le programme de formation: quel statut donner à ces activités extérieures, comment les comptabiliser, comment les rémunérer, ce qui revient à dire comment les justifier, faire en sorte qu'elles ne soient pas considérées comme des distractions en-dehors de l'apprentissage sérieux de la langue ? La première démarche de légitimation de ce travail extérieur réside certes dans les activités dont il fait l'objet en classe, aussi bien en préparation qu'en retour, comme l'ont décrit les expériences de Stauber (2009) menées autour des apprentissages alternés classe-milieu. Mais cette justification, qui vise les étudiants, doit être accompagnée de la reconnaissance de l'institution à travers le catalogue de son offre de formation et le descriptif des stages, comme sont déjà intégrées dans certains lieux les activités autonomes en centres de ressources.

\section{Conclusion}

Cette recherche-action s'attache à une évolution des pratiques d'enseignement, dans le contexte précis que sont les centres de langue en milieu homoglotte, sans remettre en cause l'existence du groupe-classe et l'intérêt d'une organisation collective des enseignements. Les activités décrites ici ont été conduites sur des cours occupant la moitié seulement du temps hebdomadaire de formation, et une partie s'intègre sans difficulté sous les intitulés de "compréhension et expression orales", de "civilisation", ou encore d'"expression écrite". Tout comme les procédures de travail en petits groupes qui ne rompent en rien avec les pratiques de cours collectif mais tendent plutôt à en renforcer l'efficacité. Il y a donc une place dans ces institutions et ces formations de type intensif et collectif pour une prise en compte des parcours individuels dans l'organisation de l'enseignement-apprentissage du français langue étrangère.

\section{BIBLIOGRAPHIE}

Abry, D., Fert, C., Parpette, C. \& Stauber, J. (2006). ICI 1. Méthode de français langue étrangère. Paris : CLE International.

Beacco, J-C., Chiss, J-L., Cicurel, F. \& Véronique, D. (2005). Les cultures éducatives et linguistiques dans l'enseignement des langues. Paris : PUF. 
Boyer, H., Butzbach, M., \& Pendanx, M. (1990). Nouvelle introduction à la didactique du français langue étrangère. Paris : CLE International.

Cuq, J.-P. (dir.). (2003). Dictionnaire de didactique du français langue étrangère et seconde. Paris : CLE International.

Dufour, S. (2007). Singularité des cours particuliers de langue étrangère : analyse cognitivointeractionnelle et pistes didactiques. Thèse de doctorat sous la direction de J.-C. Pochard. Université lumière Lyon 2.

Fievet, M. \& Abry, D. (éds). (2006). L'enseignement/ apprentissage du Français Langue Étrangère en milieu homoglotte : spécificités et exigences. Grenoble : PUG.

Parpette, C. (2006). "L'influence d'un environnement homoglotte sur l'enseignement du FLE : d'une réalité diffuse à une méthodologie constituée". in Fievet M. \& Abry D. (éds), L'enseignement/ apprentissage du Français Langue Étrangère en milieu homoglotte : spécificités et exigences. Grenoble : PUG, 21-31.

Pochard, J-C. (éd). (1994). Profils d'apprenants. Université de St Etienne.

Richterich, R. (1986). Besoins langagiers et objectifs d'apprentissage. Paris : Hachette FLE.

Stauber, J. (2009). "Un outil de découverte du milieu universitaire par les étudiants étrangers : présentation d'un protocole de mises en situation et d'analyse", in Terres de FLE 2/2009. Besançon : CLAB.

Vasseur, M.-T. (2005). Rencontres de langues, Question(s) d'interaction. Paris : Didier, LAL.

\section{NOTES}

1. Pour plus d'informations, consulter le site http://www.villagedescreateurs.com/fr/ accueil.html

2. Pour plus d'informations sur les SEL, consulter le site http://selidaire.org/spip/

3. Par exemple, Dionyssel

http://www.dailymotion.com/video/x68dd3_dionyssel_news

4. Emission de la chaine de télévision France 5.

\section{RÉSUMÉS}

Les apprenants adultes qui font la démarche de s'inscrire à un cours de FLE en France apportent avec eux leurs projets, leurs habitudes de travail, leurs goûts, qui sont autant de facteurs de diversité dans un groupe-classe. La méthodologie communicative, avec la notion d'analyse des besoins, et plus encore celle de centration sur l'apprenant, se trouve en théorie en adéquation avec cette réalité. Mais elle entre aussi en tension avec les conditions d'un enseignement collectif en milieu institutionnel. C'est à cette interface entre groupe-classe et apprenant singulier, entre classe et milieu extérieur, que se situe la recherche-action dont nous rendons compte ici, et qui se donne pour objectif d'intégrer à une méthodologie de groupe la prise en compte des profils individuels des apprenants. 
Adult learners who decide to enroll in a French as a Foreign Language Course in France bring with them their projects, their work habits and their tastes, factors which contribute to the diversity of the class. The communicative methodology, with its notion of needs analysis, and particularly that of learner-centeredness, corresponds in theory to this reality. But it also enters into conflict with the conditions of teaching to groups in an institutional setting. The field study presented in this article focuses on the interface between a class grouping and a particular pupil, between the classroom setting and the surrounding environment. Its objective is to integrate into group methodology a consideration of the individual profiles of the learners.

\section{INDEX}

Mots-clés : enseignement collectif, parcours individuels, centration sur l'apprenant, contexte homoglotte, activités extérieures

Keywords : second-language environment, group teaching, individualised learning, learercentered learning, extra-curricular activities

\section{AUTEURS}

\section{SOPHIE DUFOUR}

Sophie Dufour est docteure en Sciences du langage. Elle est enseignante de FLE au Centre International d'Études Françaises de l'université Lumière Lyon 2, à l'Institut d'Études Politiques, et de didactique à la Faculté des lettres, sciences du langage et arts.

Ses travaux de recherche portent sur la singularité et l'analyse cognitivo-interactionnelle des cours particuliers de FLE (thèse de doctorat, 2007), les pratiques interactionnelles des apprenants hors milieu guidé, et la prise en compte des parcours individuels dans l'enseignement/ apprentissage en classe.

Courriel : Sophie.Dufour[at]univ-lyon2.fr

Adresse : Centre International d'Études Françaises, 16 Quai Claude Bernard, 69365 Lyon.

\section{CHANTAL PARPETTE}

Chantal Parpette est maîtresse de conférences en didactique du FLE à l'université LumièreLyon 2. Elle partage ses activités de formation entre l'enseignement du FLE au Centre International d'Études Françaises, et la formation en didactique des étudiants du Master Français Langue Étrangère et Seconde et du Master Didactique des Langues et Tice.

Ses travaux de recherche concernent le français sur objectif spécifique, le développement des compétences académiques des étudiants et élèves allophones en France, l'analyse et l'enseignement des discours oraux, et la relation entre apprentissage formel et informel du français.

Elle est co-auteur de la méthode FLE Ici qui propose une méthodologie d'apprentissage du français articulant activités en classe et activités dans le milieu (Cle International, 2007). Elle collabore depuis 2007 à la revue Le français dans le monde dans laquelle elle rédige la rubrique Outils pour la classe. Elle est également co-auteur avec J-M Mangiante du Français sur objectif spécifique (Hachette F, 2004).

Courriel : Chantal.parpette[at]univ-lyon2.fr

Toile : http://icar.univ-lyon2.fr/membres/cparpette/ 
Adresse : Département des sciences du langage, université Lyon 2, avenue Mendès-France, 69500 Bron. 